\title{
The Multi-Attributive Border Approximation Area Comparison (MABAC) for Multiple Attribute Group Decision Making Under 2-Tuple Linguistic Neutrosophic Environment
}

\author{
Ping WANG ${ }^{1}$, Jie WANG ${ }^{2}$, Guiwu $\mathrm{WEI}^{2 *}$, Cun $\mathrm{WEI}^{2,3} \mathrm{Yu} \mathrm{WEI}^{4}$ \\ ${ }^{1}$ Institute of technology, Sichuan Normal University, Chengdu, 610101, PR China \\ ${ }^{2}$ School of Business, Sichuan Normal University, Chengdu, 610101, PR China \\ ${ }^{3}$ School of Statistics, Southwestern University of Finance and Economics, \\ Chengdu, 611130, PR China \\ ${ }^{4}$ School of Finance, Yunnan University of Finance and Economics, Kunming 650221, PR China \\ e-mail: weiguiwu1973@sicnu.edu.cn
}

Received: November 2018; accepted: April 2019

\begin{abstract}
In this paper, we present the 2-tuple linguistic neutrosophic MABAC model based on the traditional MABAC (multi-attributive border approximation area comparison) model and some fundamental theories of 2-tuple linguistic neutrosophic information. Firstly, we briefly review the definition of 2-tuple linguistic neutrosophic sets (2TLNNSs) and introduce the score function, accuracy function, operation laws and some aggregation operators of 2TLNNs. Then, the calculation steps of traditional MABAC model are briefly presented. Furthermore, combine the traditional MABAC model with 2TLNNs information, the 2-tuple linguistic neutrosophic MABAC model is established for multiple attribute group decision making (MAGDM) and the computing steps are simply depicted. In our presented model; it's more accuracy and effective for computing the distance between each alternatives and the border approximation area (BAA). Finally, a numerical example for safety assessment of construction project has been given to illustrate this new model and some comparisons between 2TLNNs MABAC model and two 2TLNNs aggregation operators are also conducted to further illustrate advantages of the new method.

Key words: multiple attribute group decision making(MAGDM), 2-tuple linguistic neutrosophic sets (2TLNSs), MABAC model, 2-tuple linguistic neutrosophic number weighted average (2TLNNWA) operators, 2-tuple linguistic neutrosophic number weighted geometric (2TLNNWG) operators, 2TLNNs MABAC model, construction project.
\end{abstract}

\section{Introduction}

The MABAC (Multi-Attributive Border Approximation area Comparison) method, which was originally defined by Pamucar and Cirovic (2015), computes the distance between each alternative and the border approximation area (BAA), and has a large amount of

\footnotetext{
*Corresponding author.
} 
unigue characteristics such as: (1) the computing results by MABAC method are stable; (2) the calculating equations are simple; (3) it takes the latent values of gains and losses into account; (4) it can be combined with other approaches. Gigovic et al. (2017) proposed the model which is based on the combined application of Geographic Information Systems (GIS) and Multi-Criteria Decision Analysis (MCDA) using the multicriteria technique of Decision Making Trial and Evaluation Laboratory (DEMATEL), the Analytic Network Process (ANP) and Multi-Attributive Border Approximation area Comparison (MABAC). Pamucar et al. (2018a) presented a new approach for the treatment of uncertainty with interval-valued fuzzy-rough numbers (IVFRN) and in this multicriteria model the traditional steps of the BWM (Best-Worst Method) and MABAC (MultiAttributive Border Approximation area Comparison) methods are modified. Pamucar $e t$ al. (2018b) presented the hybrid IR-AHP-MABAC (Interval Rough Analytic Hierarchy Process-MultiAttributive Border Approximation Area Comparison) model for evaluating the quality of university websites. Peng and Yang (2017) proposed two approaches to multiple attribute group decision making with attributes involving dependent and independent by the Pythagorean fuzzy Choquet integral average (PFCIA) operator and MABAC in Pythagorean fuzzy environment. Xue et al. (2016) proposed a novel approach based on interval-valued intuitionistic fuzzy sets (IVIFSs) and MABAC for handling material selection problems with incomplete weight information. Peng and Dai (2017a) presented three approaches to solve interval neutrosophic decision-making problems by the MABAC, evaluation based on distance from average solution (EDAS), and similarity measure. Peng and Dai (2017b) proposed three algorithms to solve hesitant fuzzy soft decision making problem by MABAC method, Weighted Aggregated Sum Product Assessment (WASPAS) and Complex Proportional Assessment (COPRAS). Peng et al. (2017a) presented three algorithms to solve interval-valued fuzzy soft decision making problems by MABAC method, Evaluation based on Distance from Average Solution (EDAS) and new similarity measure. Yu et al. (2017) developed an interval type-2 fuzzy likelihood-based MABAC approach for selecting hotels on a tourism website. Ji et al. (2018) introduced the main idea of the elimination and choice translating reality (ELECTRE) method and established an MABAC-ELECTRE method under single-valued neutrosophic linguistic environments. Peng and Dai (2018) defined some approaches to single-valued neutrosophic MADM based on MABAC, TOPSIS and new similarity measure with score function. Sharma $e t$ al. (2018) gave an efficient evaluation technique by integrating rough numbers, analytic hierarchy process (AHP) and MABAC methods in rough environment. Sun et al. (2018) established a projection-based MABAC method with hesitant fuzzy linguistic term sets (HFLTSs) and demonstrated its use in the context of patients' prioritization. Liang et al. (2019) aimed to find a suitable way to assess the risk of rockburst within complicated decision making circumstances based on the triangular fuzzy numbers (TFNs) and MABAC method. Vesković et al. (2018) proposed a new hybrid model which included a combination of the Delphi, SWARA (Step-Wise Weight Assessment Ratio Analysis) and MABAC methods for evaluation of the railway management. Bozanic et al. (2018) defined a hybrid method based on the fuzzified Analytical Hierarchical Process (AHP) method and the fuzzified MABAC method for selection of the location for deep wading as a technique 
of crossing the river by tanks. Bojanic et al. (2018) gave the hybrid model fuzzy AHPMABAC for MADM in a defensive operation of the guided anti-tank missile battery.

In previous work, lots of decision-making models such as the Best-Worst method (BWM) (Stevic et al., 2018), MultiAttributive Ideal-Real Comparative Analysis (MAIRCA) method (Chatterjee et al., 2018; Gigovic et al., 2016a; Pamucar et al., 2018c), complex proportional assessment (COPRAS) method (Bausys et al., 2015), Weighted Aggregated Sum Product Assessment (WASPAS) (Zavadskas et al., 2013), Evaluation based on Distance from Average Solution (EDAS) method (Keshavarz Ghorabaee et al., 2015), Combinative Distance-based Assessment (CODAS) method (Bolturk, 2018), Decision Making Trial and Evaluation Laboratory (DEMATEL) method (Gigovic et al., 2016b) and TODIM (an acronym in Portuguese of interactive and multiple attribute decision making) method (Gomes and Rangel, 2009; Huang and Wei, 2018; Wang et al., 2018c; Wei, 2018). Compared with the existing work, the MABAC model owns the merit of taking the distance between each alternatives and the border approximation area (BAA) into account with respect to the intangibility of decision maker (DM) and the uncertainty of decision-making environment to obtain more accuracy and effective aggregation results.

Because of the indeterminacy of DM's and the decision-making issues, we cannot always give accuracy evaluation values of alternatives to select the best project in real MADM problems. To conquer this disadvantage, fuzzy set theory which was defined by Zadeh (1965) in 1965 originally used the membership function to describe the estimation results rather than exact real numbers. Atanassov (1986) presented another measurement index which named non-membership function as a complement. Ali and Smarandache (2017) introduced the neutrosophic set (NS). Then, Wang et al. (2010) introduced the definition and some operational rules of single-valued neutrosophic sets (SVNSs). Moreover, Wang et al. (2005) extended SVNSs to interval-valued environment. Ye (2014) initially defined the single-valued neutrosophic weighted average (SVNWA) operator and singlevalued neutrosophic weighted geometric (SVNWG) operator. Wei and Wei (2018a) utilized the prioritized aggregation operators to develop some single-valued neutrosophic Dombi prioritized aggregation operators: single-valued neutrosophic Dombi prioritized average (SVNDPA) operator, single-valued neutrosophic Dombi prioritized geometric (SVNDPG) operator, single-valued neutrosophic Dombi prioritized weighted average (SVNDPWA) operator and single-valued neutrosophic Dombi prioritized weighted geometric (SVNDPWG) operator. Garg and Nancy (2018) proposed some prioritized aggregation operators based on linguistic single-valued neutrosophic (LSVN) information. Wang et al. (2018e) presented dual generalized single-valued neutrosophic number weighted Bonferroni mean (DGSVNNWBM) operator and dual generalized single-valued neutrosophic number weighted geometric Bonferroni mean (DGSVNNWGBM) operator. Liu et al. (2018) presented some Power Heronian aggregation operators based on linguistic neutrosophic environment. Xu et al. (2017) studied TODIM method under the SVN environment. Geng et al. (2018) provided some Maclaurin Symmetric Mean (MSM) Operators under interval neutrosophic linguistic information. Wu et al. (2018a) defined SVN 2-tuple linguistic sets (SVN2TLSs) and presented some new Hamacher aggregation operators. Ju et al. (2018) extended the SVN2TLSs to interval-valued environment. Wang et al. (2018d) 
defined the 2-tuple linguistic neutrosophic sets (2TLNSs) where the truth-membership function, indeterminacy-membership function and falsity-membership function are presented by 2 TLNNs. Wu et al. (2018b) proposed some Hamy mean aggregation operators of 2TLNNs. Wang et al. (2018b) proposed an extended TODIM model with 2-tuple linguistic neutrosophic information. Wang et al. (2018a) combined the original VIKOR model with a triangular fuzzy neutrosophic set to propose the triangular fuzzy neutrosophic VIKOR method. Thereafter, the SVNS has been widely investigated in MADM issues.

However, it's clear that the study about the MABAC model with 2TLNNs information does not exist. Hence, it's necessary to take 2-tuple linguistic neutrosophic MABAC model into account. The purpose of our work is to establish an extended MABAC model according to the traditional MABAC method and 2-tuple linguistic neutrosophic information to study MADM problems more effectively. Our paper is structured as: the definition, score function, accuracy function, operation rules and some aggregation operators of 2 TLNNSs are briefly introduced in section 2. The computing steps of traditional MABAC model are briefly presented in section 3 . The traditional MABAC model combined with 2TLNNs information is established, the 2-tuple linguistic neutrosophic MABAC model and the computing steps are simply depicted in Section 4 . A numerical example for safety assessment of construction project has been given to illustrate this new model and some comparisons between 2-tuple linguistic neutrosophic MABAC model and two 2TLNNs aggregation operators are also made to further illustrate advantages of the new method in Section 5. Section 6 gives some conclusions of our works.

\section{Preliminaries}

\subsection{2-Tuple Linguistic Neutrosophic Sets}

Wu et al. (2018b) initially proposed the 2-tuple linguistic neutrosophic sets (2TLNSs), which consider the unique characteristics of 2-tuple linguistic variables and single-valued neutrosophic sets (SVNSs), and can be more effective and accurate to evaluate the alternatives in multiple attribute decision making problems. To combine the 2TLSs and SVNSs, the definition of 2TLNSs can be expressed as follows.

Definition 1 (See Wu et al., 2018b). Let $\delta_{1}, \delta_{2}, \ldots, \delta_{k}$ be a linguistic term set. Any label shows a possible linguistic scale, and $\delta=\left\{\delta_{0}=\right.$ exceedingly terrible, $\delta_{1}=$ very terrible, $\delta_{2}=$ terrible, $\delta_{3}=$ medium, $\delta_{4}=$ well, $\delta_{6}=$ exceedingly well $\}$, then we can describe the 2TLNSs as:

$$
\delta=\left\langle\left(s_{t}, \alpha,\right),\left(s_{i}, \beta\right),\left(s_{f}, \chi\right)\right\rangle,
$$

where $\Delta^{-1}\left(s_{t}, \alpha,\right), \Delta^{-1}\left(s_{i}, \beta\right)$ and $\Delta^{-1}\left(s_{f}, \chi\right) \in[0, k]$ represent the truth membership function, the indeterminacy membership function and the falsity membership function which are expressed by 2 TLNNs and satisfy the condition $0 \leqslant \Delta^{-1}\left(s_{t}, \alpha\right)+\Delta^{-1}\left(s_{i}, \beta\right)+$ $\Delta^{-1}\left(s_{f}, \chi\right) \leqslant 3 k$. 
Definition 2 (See Wu et al., 2018b). Let $\delta_{1}=\left\langle\left(s_{t_{1}}, \alpha_{1},\right),\left(s_{i_{1}}, \beta_{1}\right),\left(s_{f_{1}}, \chi_{1}\right)\right\rangle$ and $\delta_{2}=$ $\left\langle\left(s_{t_{2}}, \alpha_{2},\right),\left(s_{i_{2}}, \beta_{2}\right),\left(s_{f_{2}}, \chi_{2}\right)\right\rangle$ be two 2-tuple linguistic neutrosophic numbers (2TLNNs), the operation formula of them can be defined as:

(1) $\delta_{1} \oplus \delta_{2}=\left\{\begin{array}{l}\Delta\left(k\left(\frac{\Delta^{-1}\left(s_{t_{1}}, \alpha_{1},\right)}{k}+\frac{\Delta^{-1}\left(s_{t_{2}}, \alpha_{2},\right)}{k}-\frac{\Delta^{-1}\left(s_{t_{1}}, \alpha_{1},\right)}{k} \cdot \frac{\Delta^{-1}\left(s_{t_{2}}, \alpha_{2},\right)}{k}\right)\right), \\ \Delta\left(k\left(\frac{\Delta^{-1}\left(s_{i_{1}}, \beta_{1}\right)}{k} \cdot \frac{\Delta^{-1}\left(s_{i_{2}}, \beta_{2}\right)}{k}\right)\right) \\ \Delta\left(k\left(\frac{\Delta^{-1}\left(s_{f_{1}}, \chi_{1}\right)}{k} \cdot \frac{\Delta^{-1}\left(s_{f_{1}}, \chi_{1}\right)}{k}\right)\right)\end{array}\right\}$

(2) $\delta_{1} \otimes \delta_{2}=\left\{\begin{array}{l}\Delta\left(k\left(\frac{\Delta^{-1}\left(s_{t_{1}}, \alpha_{1},\right)}{k} \cdot \frac{\Delta^{-1}\left(s_{t_{2}}, \alpha_{2},\right)}{k}\right)\right) \\ \Delta\left(k\left(\frac{\Delta^{-1}\left(s_{i_{1}}, \beta_{1}\right)}{k}+\frac{\Delta^{-1}\left(s_{i_{2}}, \beta_{2}\right)}{k}-\frac{\Delta^{-1}\left(s_{i_{1}}, \beta_{1}\right)}{k} \cdot \frac{\Delta^{-1}\left(s_{i_{2}}, \beta_{2}\right)}{k}\right)\right), \\ \Delta\left(k\left(\frac{\Delta^{-1}\left(s_{f_{1}}, \chi_{1}\right)}{k}+\frac{\Delta^{-1}\left(s_{f_{2}}, \chi_{2}\right)}{k}-\frac{\Delta^{-1}\left(s_{f_{1}}, \chi_{1}\right)}{k} \cdot \frac{\Delta^{-1}\left(s_{f_{2}}, \chi_{2}\right)}{k}\right)\right)\end{array}\right\}$

(3) $\lambda \delta_{1}=\left\{\begin{array}{l}\Delta\left(k\left(1-\left(1-\frac{\Delta^{-1}\left(s_{\left.t_{1}, \alpha_{1},\right)}\right)}{k}\right)\right)\right. \\ \Delta\left(k\left(\frac{\Delta^{-1}\left(s_{i_{1}}, \beta_{1}\right)}{k}\right)^{\lambda}\right), \\ \Delta\left(k\left(\frac{\Delta^{-1}\left(s_{f_{1}}, \chi_{1}\right)}{k}\right)^{\lambda}\right)\end{array}\right\}, \quad \lambda>0$

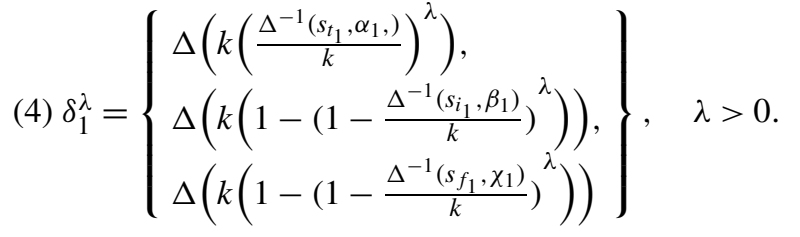

According to the Definition 2, it's clear that the operation laws have the following properties:

$$
\begin{aligned}
& \delta_{1} \oplus \delta_{2}=\delta_{2} \oplus \delta_{1}, \delta_{1} \otimes \delta_{2}=\delta_{2} \otimes \delta_{1}, \quad\left(\left(\delta_{1}\right)^{\lambda_{1}}\right)^{\lambda_{2}}=\left(\delta_{1}\right)^{\lambda_{1} \lambda_{2}}, \\
& \lambda\left(\delta_{1} \oplus \delta_{2}\right)=\lambda \delta_{1} \oplus \lambda \delta_{2}, \quad\left(\delta_{1} \otimes \delta_{2}\right)^{\lambda}=\left(\delta_{1}\right)^{\lambda} \otimes\left(\delta_{2}\right)^{\lambda}, \\
& \lambda_{1} \delta_{1} \oplus \lambda_{2} \delta_{1}=\left(\lambda_{1}+\lambda_{2}\right) \delta_{1}, \quad\left(\delta_{1}\right)^{\lambda_{1}} \otimes\left(\delta_{1}\right)^{\lambda_{2}}=\left(\delta_{1}\right)^{\left(\lambda_{1}+\lambda_{2}\right)} .
\end{aligned}
$$

Definition 3 (See Wu et al., 2018b). Let $\delta=\left\langle\left(s_{t}, \alpha,\right),\left(s_{i}, \beta\right),\left(s_{f}, \chi\right)\right\rangle$ be a 2TLNN, the score and accuracy functions of $\delta$ can be expressed as:

$$
\begin{aligned}
& s(\delta)=\frac{\left(2 k+\Delta^{-1}\left(s_{t}, \alpha,\right)-\Delta^{-1}\left(s_{i}, \beta\right)-\Delta^{-1}\left(s_{f}, \chi\right)\right)}{3 k}, \quad s(\delta) \in[0,1], \\
& h(\delta)=\frac{1}{k}\left(\Delta^{-1}\left(s_{t}, \alpha,\right)-\Delta^{-1}\left(s_{f}, \chi\right)\right), \quad h(\delta) \in[-1,1] .
\end{aligned}
$$

For two 2TLNNs $\delta_{1}$ and $\delta_{2}$, based on Definition 3, 
(1) if $s\left(\delta_{1}\right) \prec s\left(\delta_{2}\right), \quad$ then $\delta_{1} \prec \delta_{2}$;

(2) if $s\left(\delta_{1}\right) \succ s\left(\delta_{2}\right)$, then $\delta_{1} \succ \delta_{2}$;

(3) if $s\left(\delta_{1}\right)=s\left(\delta_{2}\right), h\left(\delta_{1}\right) \prec h\left(\delta_{2}\right)$, then $\delta_{1} \prec \delta_{2}$;

(4) if $s\left(\delta_{1}\right)=s\left(\delta_{2}\right), h\left(\delta_{1}\right) \succ h\left(\delta_{2}\right)$, then $\delta_{1} \succ \delta_{2}$;

(5) if $s\left(\delta_{1}\right)=s\left(\delta_{2}\right), h\left(\delta_{1}\right)=h\left(\delta_{2}\right)$, then $\delta_{1}=\delta_{2}$.

\subsection{The Distance Measurement of $2 T L N N$ s}

In the following, the normalized Hamming distance between two 2TLNNs is defined as following:

Definition 4. Let $\delta_{1}=\left\{\left(s_{t_{1}}, \alpha_{1}\right),\left(s_{i_{1}}, \beta_{1}\right),\left(s_{f_{1}}, \chi_{1}\right)\right\}$ and $\delta_{2}=\left\{\left(s_{t_{2}}, \alpha_{2}\right),\left(s_{i_{2}}, \beta_{2}\right),\left(s_{f_{2}}, \chi_{2}\right)\right\}$ be two 2 TLNNs, then we can get the normalized Hamming distance:

$$
d^{H}\left(\delta_{1}, \delta_{2}\right)=\frac{1}{3}\left(\begin{array}{l}
\left|\frac{\Delta^{-1}\left(s_{t_{1}}, \alpha_{1}\right)-\Delta^{-1}\left(s_{t_{2}}, \alpha_{2}\right)}{k}\right|+\left|\frac{\Delta^{-1}\left(s_{i_{1}}, \beta_{1}\right)-\Delta^{-1}\left(s_{i_{2}}, \beta_{2}\right)}{k}\right| \\
+\left|\frac{\Delta^{-1}\left(s_{f_{1}}, \chi_{1}\right)-\Delta^{-1}\left(s_{f_{2}}, \chi_{2}\right)}{k}\right|
\end{array}\right) .
$$

\subsection{The $2 T L N N W A$ and $2 T L N N W G$ Operators}

Wu et al. (2018b) proposed the 2-tuple linguistic neutrosophic number weighted average (2TLNNWA) operator and 2-tuple linguistic neutrosophic number weighted geometric (2TLNNWG) operator.

Definition 5 (See Wu et al., 2018b). Let $\delta_{j}=\left\{\left(s_{t_{j}}, \alpha_{j}\right),\left(s_{i_{j}}, \beta_{j}\right),\left(s_{f_{j}}, \chi_{j}\right)\right\}(j=1,2$, $\ldots, n)$ be a set of 2TLNNs, the 2TLNNWA and 2TLNNWG operators can be presented as:

$$
\begin{aligned}
& 2 \operatorname{TLNNwA}\left(\delta_{1}, \delta_{2}, \ldots, \delta_{n}\right)=w_{1} \delta_{1} \oplus w_{2} \delta_{2} \ldots \oplus w_{n} \delta_{n}=\bigoplus_{j=1}^{n} w_{j} \delta_{j} \\
& =\left\langle\begin{array}{l}
\Delta\left(k\left(1-\prod_{j=1}^{n}\left(1-\frac{\Delta^{-1}\left(s_{t_{j},}, \alpha_{j}\right)}{k}\right)^{w_{j}}\right)\right), \Delta\left(k \prod_{j=1}^{n}\left(\frac{\Delta^{-1}\left(s_{i j}, \beta_{j}\right)}{k}\right)^{w_{j}}\right), \\
\Delta\left(k \prod_{j=1}^{n}\left(\frac{\Delta^{-1}\left(s_{f_{j}}, x_{j}\right)}{k}\right)^{w_{j}}\right)
\end{array}\right\rangle
\end{aligned}
$$

and

$$
\begin{aligned}
& 2 \operatorname{TLNNWG}\left(\delta_{1}, \delta_{2}, \ldots, \delta_{n}\right)=\left(\delta_{1}\right)^{w_{1}} \otimes\left(\delta_{2}\right)^{w_{2}} \ldots \otimes\left(\delta_{n}\right)^{w_{n}}=\bigotimes_{j=1}^{n}\left(\delta_{j}\right)^{w_{j}} \\
& =\left\langle\begin{array}{l}
\Delta\left(k \prod_{j=1}^{n}\left(\frac{\Delta^{-1}\left(s_{\left.t_{j}, \alpha_{j}\right)}\right.}{k}\right)^{w_{j}}\right), \Delta\left(k\left(1-\prod_{j=1}^{n}\left(1-\frac{\Delta^{-1}\left(s_{i_{j}}, \beta_{j}\right)}{k}\right)^{w_{j}}\right)\right), \\
\Delta\left(k\left(1-\prod_{j=1}^{n}\left(1-\frac{\Delta^{-1}\left(s_{f_{j}}, \chi_{j}\right)}{k}\right)^{w_{j}}\right)\right)
\end{array}\right\rangle
\end{aligned}
$$


where $w_{j}$ is weighting vector of $\delta_{j}, j=1,2, \ldots, n$, which satisfies $0 \leqslant w_{j} \leqslant 1$, $\sum_{j=1}^{n} w_{j}=1$.

\section{The Conventional MABAC Model}

In this chapter, we will briefly review the calculating steps of the traditional MABAC model (Pamucar and Cirovic, 2015). Suppose there are $m$ alternatives $\left\{\phi_{1}, \phi_{2}, \ldots, \phi_{m}\right\}$, $n$ attributes $\left\{O_{1}, O_{2}, \ldots, O_{n}\right\}$ with weighting vector $w_{j}(j=1,2, \ldots, n)$ and $t$ experts $\left\{d_{1}, d_{2}, \ldots, d_{t}\right\}$ with weighting vector $\left\{v_{1}, v_{2}, \ldots, v_{t}\right\}$, then the decision-making steps are expressed as follows.

Step 1. Construct the evaluation matrix. $R=\left[\phi_{i j}^{t}\right]_{m \times n}, i=1,2, \ldots, m, j=1,2, \ldots, n$ which can be depicted as follows:

$$
R=\left[\phi_{i j}^{t}\right]_{m \times n}=\begin{gathered}
\mathrm{O}_{1} \\
\phi_{1} \\
\vdots \\
\phi_{m}
\end{gathered}\left[\begin{array}{llll}
\phi_{11}^{t} & \phi_{12}^{t} & \ldots & \mathrm{O}_{n} \\
\phi_{21}^{t} & \phi_{22}^{t} & \ldots & \phi_{1 n}^{t} \\
\vdots & \vdots & \vdots & \phi_{2 n}^{t} \\
\phi_{m 1}^{t} & \phi_{m 2}^{t} & \ldots & \phi_{m n}^{t}
\end{array}\right],
$$

where $\phi_{i j}^{t}(i=1,2, \ldots, m, j=1,2, \ldots, n)$ denotes the evaluation information of alternative $\phi_{i}(i=1,2, \ldots, m)$ with respect to attribute $O_{j}(j=1,2, \ldots, n)$ by expert $d^{t}$.

Step 2. According to some aggregation operators, we can utilize overall $\phi_{i j}^{t}$ to $\phi_{i j}$.

Step 3. Normalize the fused results' matrix $r=\left[\phi_{i j}\right]_{m \times n}, i=1,2, \ldots, m, j=$ $1,2, \ldots, n$ based on the type of each attributes by the following formula: For benefit attributes:

$$
N_{i j}=\phi_{i j}, \quad i=1,2, \ldots, m, j=1,2, \ldots, n
$$

For cost attributes:

$$
N_{i j}=1-\phi_{i j}, \quad i=1,2, \ldots, m, j=1,2, \ldots, n .
$$

Step 4. According to the normalized matrix $N_{i j}(i=1,2, \ldots, m, j=1,2, \ldots, n)$ and attribute's weighting vector $w_{j}(j=1,2, \ldots, n)$, the weighted normalized matrix $W N_{i j}(i=1,2, \ldots, m, j=1,2, \ldots, n)$ can be computed as:

$$
W N_{i j}=w_{j} N_{i j} \quad(i=1,2, \ldots, m, j=1,2, \ldots, n) .
$$

Step 5. Compute the values of border approximation area (BAA) and the BAA matrix $G=\left[g_{j}\right]_{1 \times n}$ can be constructed as follows:

$$
g_{j}=\left(\prod_{i=1}^{m} W N_{i j}\right)^{1 / m} \quad(i=1,2, \ldots, m, j=1,2, \ldots, n) .
$$


Step 6. Calculate the distance $D=\left[d_{i j}\right]_{m \times n}$ between each alternatives and the border approximation area (BAA) by the following equation:

$$
d_{i j}= \begin{cases}d\left(W N_{i j}, g_{j}\right), & \text { if } W N_{i j}>g_{j} \\ 0, & \text { if } W N_{i j}=g_{j} \\ -d\left(W N_{i j}, g_{j}\right) & \text { if } W N_{i j}<g_{j}\end{cases}
$$

where $d\left(W N_{i j}, g_{j}\right)$ means the distance from $W N_{i j}$ to $g_{j}$. According to the values of $d_{i j}$, we can get:

(1) if $d_{i j}>0$, the alternatives belong to the upper approximation area $G^{+}$(UAA);

(2) if $d_{i j}=0$, the alternatives belong to the border approximation area $G$ (BAA);

(3) if $d_{i j}<0$, the alternatives belong to the lower approximation area $G^{-}$(LAA).

Obviously, the best alternatives are included in $G^{+}$(UAA) and the worst alternatives are included in $G^{-}$(LAA).

Step 7. Sum the values of each alternative's $d_{i j}$ with respect to all the attributes by following equation:

$$
S_{i}=\sum_{j=1}^{n} d_{i j}
$$

According to the calculating results of $S_{i}$,we can rank all the alternatives, the bigger the value of $S_{i}$ is, the better alternative will be selected.

\section{The MABAC Model with 2-tuple Linguistic Neutrosophic Information}

By combining the MABAC method with 2-tuple linguistic neutrosophic information, we can build the 2-tuple linguistic neutrosophic MABAC model where all the evaluation information and attribute's weighting vector are presented with 2-tuple linguistic neutrosophic numbers (2TLNNs). Suppose there are $m$ alternatives $\left\{\phi_{1}, \phi_{2}, \ldots, \phi_{m}\right\}, n$ attributes $\left\{O_{1}, O_{2}, \ldots, O_{n}\right\}$ with weighting vector $w_{j}(j=1,2, \ldots, n)$ and $t$ experts $\left\{d_{1}, d_{2}, \ldots, d_{t}\right\}$ with weighting vector $\left\{v_{1}, v_{2}, \ldots, v_{t}\right\}$, the decision-making steps are expressed as follows.

Step 1. Construct the 2-tuple linguistic neutrosophic evaluation matrix $R=\left[\phi_{i j}^{t}\right]_{m \times n}$, $i=1,2, \ldots, m, j=1,2, \ldots, n$ which can be depicted as follows:

$$
R=\left[\phi_{i j}^{t}\right]_{m \times n}=\phi_{2} \phi_{1}\left[\begin{array}{cccc}
\mathrm{O}_{1} & \mathrm{O}_{2} & \ldots & \mathrm{O}_{n} \\
\phi_{11}^{t} & \phi_{12}^{t} & \ldots & \phi_{1 n}^{t} \\
\phi_{21}^{t} & \phi_{22}^{t} & \ldots & \phi_{2 n}^{t} \\
\vdots & \vdots & \vdots & \vdots \\
\phi_{m 1}^{t} & \phi_{m 2}^{t} & \ldots & \phi_{m n}^{t}
\end{array}\right]
$$


where $\phi_{i j}^{t}=\left\{\left(s_{t_{i j}}, \alpha_{i j}\right)^{t},\left(s_{i_{i j}}, \beta_{i j}\right)^{t},\left(s_{f_{i j}}, \chi_{i j}\right)^{t}\right\}(i=1,2, \ldots, m, j=1,2, \ldots, n)$ denotes the 2-tuple linguistic neutrosophic information of alternative $\phi_{i}(i=1,2, \ldots, m)$ on attribute $O_{j}(j=1,2, \ldots, n)$ by expert $d^{t}$.

Step 2. According to the 2TLNNWA or 2TLNNWG aggregation operators, we can utilize overall $\phi_{i j}^{t}$ to $\phi_{i j}$, the fused 2TLNNs matrix $r=\left[\phi_{i j}\right]_{m \times n}$ shown as follows:

$$
r=\left[\phi_{i j}\right]_{m \times n}=\begin{gathered}
\phi_{1} \\
\phi_{2} \\
\vdots \\
\phi_{m}
\end{gathered}\left[\begin{array}{cccc}
\mathrm{O}_{11} & \mathrm{O}_{2} & \ldots & \mathrm{O}_{12} \\
\phi_{21} & \phi_{22} & \ldots & \phi_{1 n} \\
\vdots & \vdots & \vdots & \vdots \\
\phi_{m 1} & \phi_{m 2} & \ldots & \phi_{m n}
\end{array}\right]
$$

where $\phi_{i j}=\left\{\left(s_{t_{i j}}, \alpha_{i j}\right),\left(s_{i_{i j}}, \beta_{i j}\right),\left(s_{i_{i j}}, \chi_{i j}\right)\right\}(i=1,2, \ldots, m, j=1,2, \ldots, n) \mathrm{de}-$ notes the fused 2-tuple linguistic neutrosophic information of alternative $\phi_{i}(i=$ $1,2, \ldots, m)$ on attribute $O_{j}(j=1,2, \ldots, n)$.

Step 3. Normalize the matrix $r=\left[\phi_{i j}\right]_{m \times n}, i=1,2, \ldots, m, j=1,2, \ldots, n$ based on the type of each attribute by the following formula; for benefit attributes:

$$
\begin{aligned}
N_{i j} & =\phi_{i j}=\left\{\left(s_{t_{i j}}, \alpha_{i j}\right)^{\prime},\left(s_{i_{i j}}, \beta_{i j}\right)^{\prime},\left(s_{f_{i j}}, \chi_{i j}\right)^{\prime}\right\} \\
& =\left\{\left(s_{t_{i j}}, \alpha_{i j}\right),\left(s_{i_{i j}}, \beta_{i j}\right),\left(s_{f_{i j}}, \chi_{i j}\right)\right\}, \\
i & =1,2, \ldots, m, j=1,2, \ldots, n .
\end{aligned}
$$

For cost attributes:

$$
\begin{aligned}
& N_{i j}=k-\phi_{i j}=\left\{\begin{array}{c}
\left(s_{t_{i j}}, \alpha_{i j}\right)^{\prime}, \\
\left(s_{i j}, \beta_{i j}\right)^{\prime}, \\
\left(s_{f_{i j}}, \chi_{i j}\right)^{\prime}
\end{array}\right\}=\left\{\begin{array}{c}
\Delta\left(k-\Delta^{-1}\left(s_{t_{i j}}, \alpha_{i j}\right)\right), \\
\Delta\left(k-\Delta^{-1}\left(s_{i_{i j}}, \beta_{i j}\right)\right), \\
\Delta\left(k-\Delta^{-1}\left(s_{f_{i j}}, \chi_{i j}\right)\right)
\end{array}\right\}, \\
& i=1,2, \ldots, m, j=1,2, \ldots, n .
\end{aligned}
$$

Step 4. According to the normalized matrix $N_{i j}=\left\{\left(s_{t i j}, \alpha_{i j}\right)^{\prime},\left(s_{i j}, \beta_{i j}\right)^{\prime},\left(s_{f_{i j}}, \chi_{i j}\right)^{\prime}\right\}$ ( $i=1,2, \ldots, m, j=1,2, \ldots, n)$ and attribute's weighting vector $w_{j}(j=1,2, \ldots, n)$, the fuzzy weighted normalized matrix $W N_{i j}=\left\{\left(s_{t_{i j}}, \alpha_{i j}\right)^{\prime \prime},\left(s_{i_{i j}}, \beta_{i j}\right)^{\prime \prime},\left(s_{f_{i j}}, \chi_{i j}\right)^{\prime \prime}\right\}$ $(i=1,2, \ldots, m, j=1,2, \ldots, n)$ can be computed as:

$$
\begin{aligned}
& W N_{i j}=w_{j} \otimes N_{i j}=\left\{\left(s_{t_{i j}}, \alpha_{i j}\right)^{\prime \prime},\left(s_{i_{i j}}, \beta_{i j}\right)^{\prime \prime},\left(s_{f_{i j}}, \chi_{i j}\right)^{\prime \prime}\right\} \\
& \quad=\left\{\begin{array}{l}
\Delta\left(k\left(1-\left(1-\frac{\Delta^{-1}\left(s_{t_{i j}}, \alpha_{i j}\right)^{\prime}}{k}\right)^{w_{j}}\right)\right), \Delta\left(k\left(\frac{\Delta^{-1}\left(s_{i_{i j}}, \beta_{i j}\right)^{\prime}}{k}\right)^{w_{j}}\right), \\
\Delta\left(k\left(\frac{\Delta^{-1}\left(s_{f_{i j}}, \chi_{i j}\right)^{\prime}}{k}\right)^{w_{j}}\right)
\end{array}\right\} \\
& (i=1,2, \ldots, m, j=1,2, \ldots, n) .
\end{aligned}
$$


Step 5. Compute the values of border approximation area (BAA) and the BAA matrix $G=\left[g_{j}\right]_{1 \times n}$ can be constructed as follows:

$$
\begin{aligned}
g_{j} & =\left(\prod_{i=1}^{m} W N_{i j}\right)^{1 / m} \\
& =\left\{\begin{array}{l}
\Delta\left(k \prod_{i=1}^{m}\left(\frac{\Delta^{-1}\left(s_{t_{i j}}, \alpha_{i j}\right)^{\prime \prime}}{k}\right)^{1 / m}\right), \\
\Delta\left(k\left(1-\prod_{i=1}^{m}\left(1-\frac{\Delta^{-1}\left(s_{i j}, \beta_{i j}\right)^{\prime \prime}}{k}\right)^{1 / m}\right)\right), \\
\Delta\left(k\left(1-\prod_{i=1}^{m}\left(1-\frac{\Delta^{-1}\left(s_{f_{i j}}, \chi_{i j}\right)^{\prime \prime}}{k}\right)^{1 / m}\right)\right)
\end{array}\right\} .
\end{aligned}
$$

Step 6. Calculate the distance $D=\left[d_{i j}\right]_{m \times n}$ between each alternatives and the border approximation area (BAA) by the following equation:

$$
d_{i j}= \begin{cases}d\left(W N_{i j}, g_{j}\right), & \text { if } W N_{i j}>g_{j}, \\ 0, & \text { if } W N_{i j}=g_{j}, \\ -d\left(W N_{i j}, g_{j}\right) & \text { if } W N_{i j}<g_{j}\end{cases}
$$

where $d\left(W N_{i j}, g_{j}\right)$ means the distance from $W N_{i j}$ to $g_{j}$.

Step 7. Sum the values of each alternative's with respect to all the attributes by the following equation:

$$
S_{i}=\sum_{j=1}^{n} d_{i j}
$$

According to the calculation results of $S_{i}$, we can rank all the alternatives, the bigger the value of $S_{i}$ is, the better alternative will be selected.

\section{The Numerical Example}

\subsection{Numerical Example for 2TLNNs MAGDM Problems}

If the construction enterprise wants to be in a good position in the new round of competition in the market, it must adapt to the changing market competition environment. In order to increase their core competitiveness, the effective method is the low cost strategy. When analysing the cost control of the construction project, it is found that there are many problems in the current cost control, especially the cost control methods are backward and rough, and the empirical elements are too many, and there is no credible basis. If you can't carry out an effective cost control, the prospects are predictable. 
Table 1

2-tuple linguistic neutrosophic evaluation information by $d^{1}$.

\begin{tabular}{lll}
\hline & $O_{1}$ & $O_{2}$ \\
\hline$\phi_{1}$ & $\left\{\left(s_{2}, 0\right),\left(s_{3}, 0\right),\left(s_{4}, 0\right)\right\}$ & $\left\{\left(s_{4}, 0\right),\left(s_{3}, 0\right),\left(s_{1}, 0\right)\right\}$ \\
$\phi_{2}$ & $\left\{\left(s_{5}, 0\right),\left(s_{1}, 0\right),\left(s_{1}, 0\right)\right\}$ & $\left\{\left(s_{5}, 0\right),\left(s_{2}, 0\right),\left(s_{1}, 0\right)\right\}$ \\
$\phi_{3}$ & $\left\{\left(s_{2}, 0\right),\left(s_{3}, 0\right),\left(s_{1}, 0\right)\right\}$ & $\left\{\left(s_{4}, 0\right),\left(s_{2}, 0\right),\left(s_{3}, 0\right)\right\}$ \\
$\phi_{4}$ & $\left\{\left(s_{4}, 0\right),\left(s_{5}, 0\right),\left(s_{4}, 0\right)\right\}$ & $\left\{\left(s_{3}, 0\right),\left(s_{4}, 0\right),\left(s_{3}, 0\right)\right\}$ \\
$\phi_{5}$ & $\left\{\left(s_{1}, 0\right),\left(s_{1}, 0\right),\left(s_{4}, 0\right)\right\}$ & $\left\{\left(s_{2}, 0\right),\left(s_{1}, 0\right),\left(s_{5}, 0\right)\right\}$ \\
\hline & $O_{3}$ & $O_{4}$ \\
\hline$\phi_{1}$ & $\left\{\left(s_{2}, 0\right),\left(s_{4}, 0\right),\left(s_{3}, 0\right)\right\}$ & $\left\{\left(s_{1}, 0\right),\left(s_{3}, 0\right),\left(s_{2}, 0\right)\right\}$ \\
$\phi_{2}$ & $\left\{\left(s_{4}, 0\right),\left(s_{1}, 0\right),\left(s_{2}, 0\right)\right\}$ & $\left\{\left(s_{5}, 0\right),\left(s_{3}, 0\right),\left(s_{4}, 0\right)\right\}$ \\
$\phi_{3}$ & $\left\{\left(s_{4}, 0\right),\left(s_{2}, 0\right),\left(s_{5}, 0\right)\right\}$ & $\left\{\left(s_{3}, 0\right),\left(s_{2}, 0\right),\left(s_{1}, 0\right)\right\}$ \\
$\phi_{4}$ & $\left\{\left(s_{2}, 0\right),\left(s_{4}, 0\right),\left(s_{1}, 0\right)\right\}$ & $\left\{\left(s_{4}, 0\right),\left(s_{5}, 0\right),\left(s_{2}, 0\right)\right\}$ \\
$\phi_{5}$ & $\left\{\left(s_{3}, 0\right),\left(s_{1}, 0\right),\left(s_{5}, 0\right)\right\}$ & $\left\{\left(s_{2}, 0\right),\left(s_{2}, 0\right),\left(s_{4}, 0\right)\right\}$ \\
\hline
\end{tabular}

Table 2

2-tuple linguistic neutrosophic evaluation information by $d^{2}$.

\begin{tabular}{lll}
\hline & $O_{1}$ & $O_{2}$ \\
\hline$\phi_{1}$ & $\left\{\left(s_{3}, 0\right),\left(s_{2}, 0\right),\left(s_{5}, 0\right)\right\}$ & $\left\{\left(s_{2}, 0\right),\left(s_{4}, 0\right),\left(s_{5}, 0\right)\right\}$ \\
$\phi_{2}$ & $\left\{\left(s_{5}, 0\right),\left(s_{2}, 0\right),\left(s_{3}, 0\right)\right\}$ & $\left\{\left(s_{5}, 0\right),\left(s_{2}, 0\right),\left(s_{3}, 0\right)\right\}$ \\
$\phi_{3}$ & $\left\{\left(s_{4}, 0\right),\left(s_{3}, 0\right),\left(s_{2}, 0\right)\right\}$ & $\left\{\left(s_{5}, 0\right),\left(s_{1}, 0\right),\left(s_{2}, 0\right)\right\}$ \\
$\phi_{4}$ & $\left\{\left(s_{3}, 0\right),\left(s_{2}, 0\right),\left(s_{5}, 0\right)\right\}$ & $\left\{\left(s_{1}, 0\right),\left(s_{5}, 0\right),\left(s_{2}, 0\right)\right\}$ \\
$\phi_{5}$ & $\left\{\left(s_{2}, 0\right),\left(s_{2}, 0\right),\left(s_{3}, 0\right)\right\}$ & $\left\{\left(s_{2}, 0\right),\left(s_{3}, 0\right),\left(s_{4}, 0\right)\right\}$ \\
\hline & $O_{3}$ & $O_{4}$ \\
\hline$\phi_{1}$ & $\left\{\left(s_{5}, 0\right),\left(s_{2}, 0\right),\left(s_{1}, 0\right)\right\}$ & $\left\{\left(s_{2}, 0\right),\left(s_{4}, 0\right),\left(s_{3}, 0\right)\right\}$ \\
$\phi_{2}$ & $\left\{\left(s_{4}, 0\right),\left(s_{3}, 0\right),\left(s_{1}, 0\right)\right\}$ & $\left\{\left(s_{4}, 0\right),\left(s_{2}, 0\right),\left(s_{1}, 0\right)\right\}$ \\
$\phi_{3}$ & $\left\{\left(s_{1}, 0\right),\left(s_{2}, 0\right),\left(s_{4}, 0\right)\right\}$ & $\left\{\left(s_{2}, 0\right),\left(s_{4}, 0\right),\left(s_{5}, 0\right)\right\}$ \\
$\phi_{4}$ & $\left\{\left(s_{5}, 0\right),\left(s_{2}, 0\right),\left(s_{3}, 0\right)\right\}$ & $\left\{\left(s_{2}, 0\right),\left(s_{4}, 0\right),\left(s_{3}, 0\right)\right\}$ \\
$\phi_{5}$ & $\left\{\left(s_{2}, 0\right),\left(s_{1}, 0\right),\left(s_{5}, 0\right)\right\}$ & $\left\{\left(s_{2}, 0\right),\left(s_{3}, 0\right),\left(s_{4}, 0\right)\right\}$ \\
\hline
\end{tabular}

Therefore, it is very important to develop and improve the cost control methods of construction projects to enhance the core competitiveness of enterprises. In order to select the best construction projects classical MADM problems are helpful (Li et al., 2018a; Li et al., 2018b; Wang et al., 2019a; Wang et al., 2019b; Wei, 2019; Wei and Zhang, 2019; Zhang et al., 2019). In this section, we provide a numerical example to select the best construction projects by using MABAC model with 2-tuple linguistic neutrosophic information. Assume that five possible construction projects $\phi_{i}(i=1,2,3,4,5)$ are to be selected and four attributes to assess these construction projects: (1) $O_{1}$ is the human factor in construction projects; (2) $\mathrm{O}_{2}$ is the energy cost factor; (3) $\mathrm{O}_{3}$ is the building materials and equipment factor; (4) $\mathrm{O}_{4}$ is the environmental factor. The five possible construction projects $\phi_{i}(i=1,2,3,4,5)$ are to be evaluated with 2TLNNs with the four criteria by three experts $d^{t}$ (Suppose expert's weighting vector is $(0.3,0.4,0.3)$ and attribute's weighting vector is $(0.4,0.1,0.3,0.2))$.

Step 1. Construct the 2-tuple linguistic neutrosophic evaluation matrix $R=\left[\phi_{i j}^{t}\right]_{m \times n}$, $i=1,2, \ldots, m, j=1,2, \ldots, n$. 
Table 3

2-tuple linguistic neutrosophic evaluation information by $d^{3}$.

\begin{tabular}{lll}
\hline & $O_{1}$ & $O_{2}$ \\
\hline$\phi_{1}$ & $\left\{\left(s_{2}, 0\right),\left(s_{1}, 0\right),\left(s_{4}, 0\right)\right\}$ & $\left\{\left(s_{1}, 0\right),\left(s_{3}, 0\right),\left(s_{5}, 0\right)\right\}$ \\
$\phi_{2}$ & $\left\{\left(s_{4}, 0\right),\left(s_{1}, 0\right),\left(s_{2}, 0\right)\right\}$ & $\left\{\left(s_{5}, 0\right),\left(s_{2}, 0\right),\left(s_{1}, 0\right)\right\}$ \\
$\phi_{3}$ & $\left\{\left(s_{5}, 0\right),\left(s_{4}, 0\right),\left(s_{4}, 0\right)\right\}$ & $\left\{\left(s_{2}, 0\right),\left(s_{5}, 0\right),\left(s_{4}, 0\right)\right\}$ \\
$\phi_{4}$ & $\left\{\left(s_{5}, 0\right),\left(s_{3}, 0\right),\left(s_{4}, 0\right)\right\}$ & $\left\{\left(s_{1}, 0\right),\left(s_{4}, 0\right),\left(s_{3}, 0\right)\right\}$ \\
$\phi_{5}$ & $\left\{\left(s_{1}, 0\right),\left(s_{2}, 0\right),\left(s_{4}, 0\right)\right\}$ & $\left\{\left(s_{4}, 0\right),\left(s_{2}, 0\right),\left(s_{3}, 0\right)\right\}$ \\
\hline & $O_{3}$ & $O_{4}$ \\
\hline$\phi_{1}$ & $\left\{\left(s_{3}, 0\right),\left(s_{4}, 0\right),\left(s_{2}, 0\right)\right\}$ & $\left\{\left(s_{3}, 0\right),\left(s_{5}, 0\right),\left(s_{2}, 0\right)\right\}$ \\
$\phi_{2}$ & $\left\{\left(s_{4}, 0\right),\left(s_{1}, 0\right),\left(s_{2}, 0\right)\right\}$ & $\left\{\left(s_{5}, 0\right),\left(s_{3}, 0\right),\left(s_{2}, 0\right)\right\}$ \\
$\phi_{3}$ & $\left\{\left(s_{3}, 0\right),\left(s_{2}, 0\right),\left(s_{5}, 0\right)\right\}$ & $\left\{\left(s_{1}, 0\right),\left(s_{3}, 0\right),\left(s_{4}, 0\right)\right\}$ \\
$\phi_{4}$ & $\left\{\left(s_{2}, 0\right),\left(s_{4}, 0\right),\left(s_{1}, 0\right)\right\}$ & $\left\{\left(s_{3}, 0\right),\left(s_{5}, 0\right),\left(s_{2}, 0\right)\right\}$ \\
$\phi_{5}$ & $\left\{\left(s_{2}, 0\right),\left(s_{3}, 0\right),\left(s_{4}, 0\right)\right\}$ & $\left\{\left(s_{4}, 0\right),\left(s_{2}, 0\right),\left(s_{1}, 0\right)\right\}$ \\
\hline
\end{tabular}

Table 4

The aggregation results by 2 TLNNWA operator.

\begin{tabular}{lll}
\hline & $O_{1}$ & $O_{2}$ \\
\hline$\phi_{1}$ & $\left\{\left(s_{2}, 0.4348\right),\left(s_{2},-0.1654\right),\left(s_{4}, 0.3734\right)\right\}$ & $\left\{\left(s_{3},-0.4740\right),\left(s_{3}, 0.3659\right),\left(s_{3}, 0.0852\right)\right\}$ \\
$\phi_{2}$ & $\left\{\left(s_{5},-0.2311\right),\left(s_{1}, 03195\right),\left(s_{2},-0.0895\right)\right\}$ & $\left\{\left(s_{1}, 0.4269\right),\left(s_{2}, 0.3522\right),\left(s_{5}, 0.0000\right)\right\}$ \\
$\phi_{3}$ & $\left\{\left(s_{4}, 0.0000\right),\left(s_{3}, 0.2704\right),\left(s_{2}, 0.0000\right)\right\}$ & $\left\{\left(s_{4}, 0.1339\right),\left(s_{2},-0.0047\right),\left(s_{3},-0.2192\right)\right\}$ \\
$\phi_{4}$ & $\left\{\left(s_{4}, 0.0895\right),\left(s_{3},-0.0267\right),\left(s_{4}, 0.3734\right)\right\}$ & $\left\{\left(s_{2},-0.2896\right),\left(s_{4}, 0.3734\right),\left(s_{3},-0.4492\right)\right\}$ \\
$\phi_{5}$ & $\left\{\left(s_{1}, 0.4269\right),\left(s_{2},-0.3755\right),\left(s_{4},-0.4348\right)\right\}$ & $\left\{\left(s_{3},-0.2490\right),\left(s_{2},-0.0895\right),\left(s_{4},-0.0767\right)\right\}$ \\
\hline & $O_{3}$ & $O_{4}$ \\
\hline$\phi_{1}$ & $\left\{\left(s_{4},-0.1074\right),\left(s_{3}, 0.0314\right),\left(s_{2},-0.2882\right)\right\}$ & $\left\{\left(s_{2}, 0.0767\right),\left(s_{4},-0.0767\right),\left(s_{2}, 0.3522\right)\right\}$ \\
$\phi_{2}$ & $\left\{\left(s_{4}, 0.0000\right),\left(s_{2},-0.4482\right),\left(s_{2},-0.4843\right)\right\}$ & $\left\{\left(s_{5},-0.3195\right),\left(s_{3},-0.4492\right),\left(s_{2},-0.1339\right)\right\}$ \\
$\phi_{3}$ & $\left\{\left(s_{3},-0.2586\right),\left(s_{2}, 0.0000\right),\left(s_{5},-0.4269\right)\right\}$ & $\left\{\left(s_{2}, 0.0767\right),\left(s_{3},-0.0196\right),\left(s_{3},-0.1146\right)\right\}$ \\
$\phi_{4}$ & $\left\{\left(s_{4},-0.2974\right),\left(s_{3}, 0.0314\right),\left(s_{2},-0.4482\right)\right\}$ & $\left\{\left(s_{3}, 0.0196\right),\left(s_{5},-0.4269\right),\left(s_{2}, 0.3522\right)\right\}$ \\
$\phi_{5}$ & $\left\{\left(s_{2}, 0.3307\right),\left(s_{1}, 0.3904\right),\left(s_{5},-0.3238\right)\right\}$ & $\left\{\left(s_{3},-0.2490\right),\left(s_{2}, 0.3522\right),\left(s_{3},-0.3610\right)\right\}$ \\
\hline
\end{tabular}

Step 2. Then according to 2 TLNNWA operator and expert's weighting vector, we can utilize overall $\phi_{i j}^{t}$ to $\phi_{i j}$ to obtain the matrix $r=\left[\phi_{i j}\right]_{m \times n}, i=1,2, \ldots, m, j=$ $1,2, \ldots, n$ as follows.

Step 3. Normalize the fused results matrix $r=\left[\phi_{i j}\right]_{m \times n}, i=1,2, \ldots, m, j=1,2, \ldots, n$ based on the type of each attributes by formula (19) and (20); (O2 is the cost attribute).

Step 4. According to the normalized matrix $(i=1,2, \ldots, m, j=1,2, \ldots, n)$ and attribute's weighting vector $w_{j}(j=1,2, \ldots, n)$, the weighted normalized matrix $W N_{i j}, i=1,2, \ldots, m, j=1,2, \ldots, n$ can be computed as:

Step 5. Compute the values of border approximation area (BAA) and the BAA matrix $G=\left[g_{j}\right]_{1 \times n}$ can be constructed as follows:

$$
\begin{aligned}
& g_{1}=\left\{\left(s_{2},-1.6327\right)\left(s_{4},-3.2448\right),\left(s_{5},-4.1188\right)\right\}, \\
& g_{2}=\left\{\left(s_{0}, 0.1179,\right)\left(s_{6},-5.1224\right),\left(s_{6},-5.0539\right)\right\}, \\
& g_{3}=\left\{\left(s_{1},-0.7498\right)\left(s_{4},-3.1852\right),\left(s_{5},-4.3335\right)\right\},
\end{aligned}
$$


Table 5

The normalized decision-making matrix $N_{i j}$.

\begin{tabular}{lll}
\hline & $O_{1}$ & $O_{2}$ \\
\hline$\phi_{1}$ & $\left\{\left(s_{2}, 0.4348\right),\left(s_{2},-0.1654\right),\left(s_{4}, 0.3734\right)\right\}$ & $\left\{\left(s_{3}, 0.4740\right),\left(s_{3},-0.3659\right),\left(s_{3},-0.0852\right)\right\}$ \\
$\phi_{2}$ & $\left\{\left(s_{5},-0.2311\right),\left(s_{1}, 0.3195\right),\left(s_{2},-0.0895\right)\right\}$ & $\left\{\left(s_{5},-0.4269\right),\left(s_{4},-0.3522\right),\left(s_{1}, 0.0000\right)\right\}$ \\
$\phi_{3}$ & $\left\{\left(s_{4}, 0.0000\right),\left(s_{3}, 0.2704\right),\left(s_{2}, 0.0000\right)\right\}$ & $\left\{\left(s_{2},-0.1339\right),\left(s_{4}, 0.0047\right),\left(s_{3}, 0.2192\right)\right\}$ \\
$\phi_{4}$ & $\left\{\left(s_{4}, 0.0895\right),\left(s_{3},-0.2367\right),\left(s_{4}, 0.3734\right)\right\}$ & $\left\{\left(s_{4}, 0.2896\right),\left(s_{2},-0.3734\right),\left(s_{3}, 0.4492\right)\right\}$ \\
$\phi_{5}$ & $\left\{\left(s_{1}, 0.4269\right),\left(s_{2},-0.3755\right),\left(s_{4},-0.4348\right)\right\}$ & $\left\{\left(s_{3}, 0.2490\right),\left(s_{4}, 0.0895\right),\left(s_{2}, 0.0767\right)\right\}$ \\
\hline & $O_{3}$ & $O_{4}$ \\
\hline$\phi_{1}$ & $\left\{\left(s_{4},-0.1074\right),\left(s_{3}, 0314\right),\left(s_{2},-0.2882\right)\right\}$ & $\left\{\left(s_{2}, 0767\right),\left(s_{4},-0.0767\right),\left(s_{2}, 0.3522\right)\right\}$ \\
$\phi_{2}$ & $\left\{\left(s_{4}, 0.0000\right),\left(s_{2},-0.4482\right),\left(s_{2},-0.4843\right)\right\}$ & $\left\{\left(s_{5},-0.3195\right),\left(s_{3},-0.4492\right),\left(s_{2},-0.1339\right)\right\}$ \\
$\phi_{3}$ & $\left\{\left(s_{3},-0.2586\right),\left(s_{2}, 0.0000\right),\left(s_{5},-0.4269\right)\right\}$ & $\left\{\left(s_{2}, 0.0767\right),\left(s_{3},-0.0196\right),\left(s_{3},-0.1146\right)\right\}$ \\
$\phi_{4}$ & $\left\{\left(s_{4},-0.2974\right),\left(s_{3}, 0.0314\right),\left(s_{2},-0.4482\right)\right\}$ & $\left\{\left(s_{3}, 0.0196\right),\left(s_{5},-0.4269\right),\left(s_{2}, 0.3522\right)\right\}$ \\
$\phi_{5}$ & $\left\{\left(s_{2}, 0.3307\right),\left(s_{1}, 0.3904\right),\left(s_{5},-0.3238\right)\right\}$ & $\left\{\left(s_{3},-0.2490\right),\left(s_{2}, 0.3522\right),\left(s_{3},-0.3610\right)\right\}$ \\
\hline
\end{tabular}

Table 6

Weighted normalized average matrix $W N_{i j}$.

\begin{tabular}{lll}
\hline & $O_{1}$ & $O_{2}$ \\
\hline$\phi_{1}$ & $\left\{\left(s_{1}, 0.1278\right),\left(s_{4},-0.2648\right),\left(s_{5}, 0.2871\right)\right\}$ & $\left\{\left(s_{0}, 0.4972\right),\left(s_{6},-0.4741\right),\left(s_{6},-0.4179\right)\right\}$ \\
$\phi_{2}$ & $\left\{\left(s_{3},-0.1843\right),\left(s_{3}, 0.2738\right),\left(s_{4},-0.2038\right)\right\}$ & $\left\{\left(s_{1},-0.1973\right),\left(s_{6},-0.2913\right),\left(s_{5}, 0.0158\right)\right\}$ \\
$\phi_{3}$ & $\left\{\left(s_{2}, 0.1336\right),\left(s_{5},-0.2931\right),\left(s_{4},-0.1336\right)\right\}$ & $\left\{\left(s_{0}, 0.2194\right),\left(s_{6},-0.2377\right),\left(s_{2}, 3622\right)\right\}$ \\
$\phi_{4}$ & $\left\{\left(s_{2}, 0.2038\right),\left(s_{5},-0.4691\right),\left(s_{5}, 0.2871\right)\right\}$ & $\left\{\left(s_{1},-0.2923\right),\left(s_{5}, 0.2658\right),\left(s_{6},-0.3232\right)\right\}$ \\
$\phi_{5}$ & $\left\{\left(s_{1},-0.3824\right),\left(s_{4},-0.4422\right),\left(s_{5},-0.1278\right)\right\}$ & $\left\{\left(s_{0}, 0.4501\right),\left(s_{6},-0.2257\right),\left(s_{5}, 0.3960\right)\right\}$ \\
\hline & $O_{3}$ & $O_{4}$ \\
\hline$\phi_{1}$ & $\left\{\left(s_{3},-0.3836\right),\left(s_{5},-0.1112\right),\left(s_{4}, 0.1185\right)\right\}$ & $\left\{\left(s_{0}, 0.4887\right),\left(s_{6},-0.4887\right),\left(s_{5},-0.0248\right)\right\}$ \\
$\phi_{2}$ & $\left\{\left(s_{2},-0.3153\right),\left(s_{4},-0.0009\right),\left(s_{4},-0.0291\right)\right\}$ & $\left\{\left(s_{2},-0.4320\right),\left(s_{5}, 0.0566\right),\left(s_{5}, 0.2499\right)\right\}$ \\
$\phi_{3}$ & $\left\{\left(s_{1}, 0.0041\right),\left(s_{4}, 0.3153\right),\left(s_{6},-0.4695\right)\right\}$ & $\left\{\left(s_{0}, 0.4887\right),\left(s_{5}, 0.2164\right),\left(s_{5}, 0.1828\right)\right\}$ \\
$\phi_{4}$ & $\left\{\left(s_{2},-0.4986\right),\left(s_{5},-0.1112\right),\left(s_{4},-0.0009\right)\right\}$ & $\left\{\left(s_{1},-0.2164\right),\left(s_{6},-0.3172\right),\left(s_{5},-0.0248\right)\right\}$ \\
$\phi_{5}$ & $\left\{\left(s_{1},-0.1770\right),\left(s_{4},-0.1306\right),\left(s_{6},-0.4323\right)\right\}$ & $\left\{\left(s_{1},-0.3073\right),\left(s_{5},-0.0248\right),\left(s_{5}, 0.0911\right)\right\}$ \\
\hline
\end{tabular}

Table 7

The distances between $d_{i j}$ alternatives and BAA.

\begin{tabular}{lllll}
\hline & $O_{1}$ & $O_{2}$ & $O_{3}$ & $O_{4}$ \\
\hline$\phi_{1}$ & $\{-0.0693\}$ & $\{0.0114\}$ & $\{0.0870\}$ & $\{-0.0239\}$ \\
$\phi_{2}$ & $\{0.1673\}$ & $\{0.0481\}$ & $\{0.1000\}$ & $\{0.0771\}$ \\
$\phi_{3}$ & $\{0.1195\}$ & $\{-0.0286\}$ & $\{-0.0573\}$ & $\{-0.0305\}$ \\
$\phi_{4}$ & $\{-0.0910\}$ & $\{0.0428\}$ & $\{0.0873\}$ & $\{-0.0234\}$ \\
$\phi_{5}$ & $\{-0.0844\}$ & $\{-0.0154\}$ & $\{-0.0942\}$ & $\{0.0274\}$ \\
\hline
\end{tabular}

$$
g_{4}=\left\{\left(s_{1},-0.8694\right),\left(s_{5},-4.0529\right),\left(s_{5},-4.1708\right)\right\}
$$

Step 6. Calculate the distance $D=\left[d_{i j}\right]_{m \times n}$ between each alternatives and the border approximation area (BAA) by equation (23).

Step 7. Sum the values of each alternative's $d_{i j}$ with respect to all the attributes by equation (24);

$$
S_{1}=0.0052, \quad S_{2}=0.3925, \quad S_{3}=0.0032, \quad S_{4}=0.0157, \quad S_{5}=-0.1665 .
$$


Table 8

The fused values by using some 2 TLNNs aggregation operator.

\begin{tabular}{lll}
\hline & 2TLNNWA & 2TLNNWG \\
\hline$\phi_{1}$ & $\left\{\left(s_{3},-0.3685\right),\left(s_{3},-0.3615\right),\left(s_{3},-0.1843\right)\right\}$ & $\left\{\left(s_{3},-0.2749\right),\left(s_{3},-0.1273\right),\left(s_{3}, 0.2894\right)\right\}$ \\
$\phi_{2}$ & $\left\{\left(s_{4},-0.1286\right),\left(s_{2},-0.3254\right),\left(s_{2},-0.0469\right)\right\}$ & $\left\{\left(s_{4},-0.0053\right),\left(s_{2},-0.2298\right),\left(s_{2}, 0.3403\right)\right\}$ \\
$\phi_{3}$ & $\left\{\left(s_{3},-0.1443\right),\left(s_{3},-0.3636\right),\left(s_{3},-0.1494\right)\right\}$ & $\left\{\left(s_{3}, 0.1429\right),\left(s_{3},-0.2457\right),\left(s_{3}, 0.2672\right)\right\}$ \\
$\phi_{4}$ & $\left\{\left(s_{3}, 0.1191\right),\left(s_{3}, 0.3878\right),\left(s_{3},-0.3175\right)\right\}$ & $\left\{\left(s_{3}, 0.4239\right),\left(s_{4},-0.4331\right),\left(s_{3}, 0.2130\right)\right\}$ \\
$\phi_{5}$ & $\left\{\left(s_{2},-0.0375\right),(s 2,-0.3032),\left(s_{4},-0.3233\right)\right\}$ & $\left\{(s 2,0.0131),(s 2,-0.2568),\left(s_{4},-0.1289\right)\right\}$ \\
\hline
\end{tabular}

Table 9

Score results of alternatives $\phi_{i}$.

\begin{tabular}{lll}
\hline & 2TLNNWA & 2TLNNWG \\
\hline$s\left(\phi_{1}\right)$ & 0.6663 & 0.6585 \\
$s\left(\phi_{2}\right)$ & 0.7877 & 0.7902 \\
$s\left(\phi_{3}\right)$ & 0.6789 & 0.6883 \\
$s\left(\phi_{4}\right)$ & 0.6517 & 0.6587 \\
$s\left(\phi_{5}\right)$ & 0.6814 & 0.6817 \\
\hline
\end{tabular}

Table 10

Rank of alternatives by some 2TLNNs aggregation operators.

\begin{tabular}{ll}
\hline & Order \\
\hline 2TLNNWA & $\left\{\phi_{2}>\phi_{5}>\phi_{3}>\phi_{1}>\phi_{4}\right\}$ \\
2TLNNWG & $\left\{\phi_{2}>\phi_{3}>\phi_{5}>\phi_{4}>\phi_{1}\right\}$ \\
2TLNNs MABAC model & $\left\{\phi_{2}>\phi_{4}>\phi_{1}>\phi_{3}>\phi_{5}\right\}$ \\
\hline
\end{tabular}

According to the calculating results of $S_{i}$, we can rank all the alternatives, the bigger the value of $S_{i}$ is, the better alternative will be selected. Obviously, the rank of all alternatives is $\phi_{2}>\phi_{4}>\phi_{1}>\phi_{3}>\phi_{5}$ and $\phi_{2}$ is the best alternative.

\subsection{Comparison of 2TLNNs MABAC Method with Some 2TLNNs Aggregation Operators}

In this chapter, we compare our proposed 2-tuple linguistic neutrosophic MABAC method with the 2-tuple linguistic neutrosophic weighted average (2TLNNWA) operator and the 2-tuple linguistic neutrosophic weighted geometric (2TLNNWG) operator (Wu et al., 2018b). Based on the attribute's weight and results of Table 4, the fused values by 2TLNNWA and 2TLNNWG operators are shown in Table 8.

According to the score function of 2 TLNNs, we can obtain the alternative score results which are shown in Table 9.

The ranking of alternatives by some 2 TLNNs aggregation operators are listed as follows.

Comparing the results of the 2-tuple linguistic neutrosophic MABAC model with 2TLNNWA and 2TLNNWG operators, the aggregation results are slightly different in ranking of alternatives and the best alternatives are same. However, 2-tuple linguistic 
neutrosophic MABAC model has the unique characteristics of computing the distance between each alternatives and the border approximation area (BAA) and can be more accurate and effective in the application of MADM problems.

\section{Conclusions}

In this paper, we present the 2-tuple linguistic neutrosophic MABAC model based on the traditional MABAC (multi-attributive border approximation area comparison) model and some fundamental theories of 2-tuple linguistic neutrosophic information. Firstly, we briefly review the definition of 2-tuple linguistic neutrosophic sets (2TLNNSs) and introduce the score function, accuracy function, operation laws and some aggregation operators of 2TLNNs. Then, the calculation steps of traditional MABAC model are briefly presented. Furthermore, by combining the traditional MABAC model with 2TLNNs information, the 2-tuple linguistic neutrosophic MABAC model is established and the computing steps are simply depicted. Our presented model is more accurate and effective for computing the distance between each alternatives and the border approximation area (BAA). Finally, a numerical example for safety assessment of construction project has been given to illustrate this new model and some comparisons between 2TLNNs MABAC model and two 2TLNNs aggregation operators are also made to further illustrate the advantages of the new method. In the future, the 2-tuple linguistic neutrosophic MABAC model can be applied to the risk analysis (Khraisha and Arthur, 2018; Lenka and Barik, 2018; Wei et al., 2017; Wei et al., 2018f), the MADM problems (Dong et al., 2018; Kou et al., 2016; Mardani et al., 2018; Morente-Molinera et al., 2018; Sremac et al., 2018) and many other uncertain and fuzzy environments (Ghorabaee et al., 2017; Peng et al., 2018; Peng and Garg, 2018; Peng and Yang, 2017; Peng et al., 2017b; Wei and Gao, 2018; Wei et al., 2018a, 2018b, 2018c, 2018d, 2018e; Wei et al., 2019; Wei and Wei, 2018b)

\section{References}

Ali, M., Smarandache, F. (2017). Complex neutrosophic set. Neural Computing \& Applications, 28, 1817-1834. Atanassov, K.T. (1986). Intuitionistic fuzzy sets. Fuzzy Sets and Systems, 20, 87-96.

Bausys, R., Zavadskas, E.K., Kaklauskas, A. (2015). Application of neutrosophic set to multicriteria decision making by COPRAS. Economic Computation and Economic Cybernetics Studies and Research, 49, 91-105.

Bojanic, D., Kovac, M., Bojanic, M., Ristic, V. (2018). Multi-criteria decision-making in a defensive operation of the guided anti-tank missile battery: An example of the hybrid model fuzzy AHP-MABAC. Decision Making: Applications in Management and Engineering, 1, 51-66.

Bolturk, E. (2018). Pythagorean fuzzy CODAS and its application to supplier selection in a manufacturing firm. Journal of Enterprise Information Management, 31, 550-564.

Bozanic, D., Tešić, D., Milićević, J. (2018). A hybrid fuzzy AHP-MABAC model: application in the Serbian Arm-The selection of the location for deep wading as a technique of crossing the river by tanks. Decision Making: Applications in Management and Engineering, 1, 143-164.

Chatterjee, K., Pamucar, D., Zavadskas, E.K. (2018). Evaluating the performance of suppliers based on using the R'AMATEL-MAIRCA method for green supply chain implementation in electronics industry. Journal of Cleaner Production, 184, 101-129. 
Dong, Y.C., Zha, Q.B., Zhang, H.J., Kou, G., Fujita, H., Chiclana, F., Herrera-Viedma, E. (2018). Consensus reaching in social network group decision making: Research paradigms and challenges. Knowledge-Based Systems, 162, 3-13.

Garg, H., Nancy. (2018). Linguistic single-valued neutrosophic prioritized aggregation operators and their applications to multiple-attribute group decision-making. Journal of Ambient Intelligence and Humanized Computing, 9, 1975-1997.

Geng, Y.H., Wang, X.G., Li, X.M., Yu, K., Liu, P.D. (2018). Some interval neutrosophic linguistic maclaurin symmetric mean operators and their application in multiple attribute decision making. Symmetry-Basel, 10.

Ghorabaee, M.K., Amiri, M., Zavadskas, E.K., Antuchevičienė, J.J.T. (2017). Assessment of third-party logistics providers using a CRITIC-WASPAS approach with interval type-2 fuzzy sets. Transpot, 32, 66-78.

Gigovic, L.J., Pamucar, D., Bajic, Z., Milicevic, M. (2016a). The combination of expert judgment and GISMAIRCA analysis for the selection of sites for ammunition depots. Sustainability, 8.

Gigovic, L.J., Pamucar, D., Lukic, D., Markovic, S. (2016b). GIS-Fuzzy DEMATEL MCDA model for the evaluation of the sites for ecotourism development: a case study of "Dunayski Kljuc" region, Serbia. Land Use Policy, 58, 348-365.

Gigovic, L., Pamucar, D., Bozanic, D., Ljubojevic, S. (2017). Application of the GIS-DANP-MABAC multicriteria model for selecting the location of wind farms: A case study of Vojvodina, Serbia. Renewable Energy, 103, 501-521.

Gomes, L., Rangel, L.A.D. (2009). An application of the TODIM method to the multicriteria rental evaluation of residential properties. European Journal of Operational Research, 193, 204-211.

Huang, Y.H., Wei, G.W. (2018). TODIM method for Pythagorean 2-tuple linguistic multiple attribute decision making. Journal of Intelligent \& Fuzzy Systems, 35, 901-915.

Ji, P., Zhang, H.Y., Wang, J.Q. (2018). Selecting an outsourcing provider based on the combined MABACELECTRE method using single-valued neutrosophic linguistic sets. Computers \& Industrial Engineering, 120, 429-441.

Ju, D.W., Ju, Y.B., Wang, A.H. (2018). Multiple attribute group decision making based on Maclaurin symmetric mean operator under single-valued neutrosophic interval 2-tuple linguistic environment. Journal of Intelligent \& Fuzzy Systems, 34, 2579-2595.

Keshavarz Ghorabaee, M., Zavadskas, E.K., Olfat, L., Turskis, Z. (2015). Multi-criteria inventory classification using a new method of evaluation based on distance from average solution (EDAS). Informatica, 26, 435451 .

Khraisha, T., Arthur, K. (2018). Can we have a general theory of financial innovation processes? A conceptual review. Financial Innovation, 4.

Kou, G., Ergu, D., Lin, C.S., Chen, Y. (2016). Pairwise comparison matrix in multiple criteria decision making. Technological and Economic Development of Economy, 22, 738-765.

Lenka, S.K., Barik, R. (2018). Has expansion of mobile phone and internet use spurred financial inclusion in the SAARC countries? Financial Innovation, 4.

Li, Z.X., Gao, H., Wei, G.W. (2018a). Methods for multiple attribute group decision making based on intuitionistic fuzzy dombi hamy mean operators. Symmetry-Basel, 10, 574.

Li, Z.X., Wei, G.W., Gao, H. (2018b). Methods for multiple attribute decision making with interval-valued Pythagorean fuzzy information. Mathematics, 6, 228.

Liang, W.Z., Zhao, G.Y., Wu, H., Dai, B. (2019). Risk assessment of rockburst via an extended MABAC method under fuzzy environment. Tunnelling and Underground Space Technology, 83, 533-544.

Liu, P.D., Mahmood, T., Khan, Q. (2018). Group decision making based on power heronian aggregation operators under linguistic neutrosophic environment. International Journal of Fuzzy Systems, 20, 970-985.

Mardani, A., Nilashi, M., Zavadskas, E.K., Awang, S.R., Zare, H., Jamal, N.M. (2018). Decision making methods based on fuzzy aggregation operators: three decades review from 1986 to 2017. International Journal of Information Technology \& Decision Making, 17, 391-466.

Morente-Molinera, J.A., Kou, G., Peng, Y., Torres-Albero, C., Herrera-Viedma, E. (2018). Analysing discussions in social networks using group decision making methods and sentiment analysis. Information Sciences, 447 , $157-168$.

Pamucar, D., Cirovic, G. (2015). The selection of transport and handling resources in logistics centers using multi-attributive border approximation area comparison (MABAC). Expert Systems with Applications, 42, 3016-3028.

Pamucar, D., Petrovic, I., Cirovic, G. (2018a). Modification of the best-worst and MABAC methods: a novel approach based on interval-valued fuzzy-rough numbers. Expert Systems with Applications, 91, 89-106. 
Pamucar, D., Stevic, Z., Zavadskas, E.K. (2018b). Integration of interval rough AHP and interval rough MABAC methods for evaluating university web pages. Applied Soft Computing, 67, 141-163.

Pamucar, D.S., Tarle, S.P., Parezanovic, T. (2018c). New hybrid multi-criteria decision-making DEMATELMAIRCA model: sustainable selection of a location for the development of multimodal logistics centre. Economic Research-Ekonomska Istrazivanja, 31, 1641-1665.

Peng, X.D., Dai, J.G. (2017a). Algorithms for interval neutrosophic multiple attribute decision-making based on MABAC, similarity measure, and EDAS. International Journal for Uncertainty Quantification, 7, 395-421.

Peng, X.D., Dai, J.G. (2017b). Hesitant fuzzy soft decision making methods based on WASPAS, MABAC and COPRAS with combined weights. Journal of Intelligent \& Fuzzy Systems, 33, 1313-1325.

Peng, X.D., Dai, J.G. (2018). Approaches to single-valued neutrosophic MADM based on MABAC, TOPSIS and new similarity measure with score function. Neural Computing \& Applications, 29, 939-954.

Peng, X.D., Yang, Y. (2017). Algorithms for interval-valued fuzzy soft sets in stochastic multi-criteria decision making based on regret theory and prospect theory with combined weight. Applied Soft Computing, 54, 415-430.

Peng, X.D., Garg, H. (2018). Algorithms for interval-valued fuzzy soft sets in emergency decision making based on WDBA and CODAS with new information measure. Computers \& Industrial Engineering, 119, 439-452.

Peng, X.D., Dai, J.G., Yuan, H.Y. (2017a). Interval-valued fuzzy soft decision making methods based on MABAC, similarity measure and EDAS. Fundamenta Informaticae, 152, 373-396.

Peng, X.D., Yuan, H.Y., Yang, Y. (2017b). Pythagorean fuzzy information measures and their applications. International Journal of Intelligent Systems, 32, 991-1029.

Peng, X.D., Dai, J.G., Liu, L. (2018). Interval-valued dual hesitant fuzzy information aggregation and its application in multiple attribute decision making. International Journal for Uncertainty Quantification, 8, 361-382.

Sharma, H.K., Roy, J., Kar, S., Prentkovskis, O. (2018). Multi criteria evaluation framework for prioritizing indian railway stations using modified rough AHP-MABAC method. Transport and Telecommunication Journal, 19, 113-127.

Sremac, S., Stevic, Z., Pamucar, D., Arsic, M., Matic, B. (2018). Evaluation of a third-party logistics (3PL) provider using a rough SWARA-WASPAS model based on a new rough dombi aggregator. Symmetry-Basel, 10,305 .

Stevic, Z., Pamucar, D., Subotic, M., Antucheviciene, J., Zavadskas, E.K. (2018). The location selection for roundabout construction using rough BWM-rough WASPAS approach based on a new rough hamy aggregator. Sustainability, 10

Sun, R.X., Hu, J.H., Zhou, J.D., Chen, X.H. (2018). A hesitant fuzzy linguistic projection-based MABAC method for patients' prioritization. International Journal of Fuzzy Systems, 20, 2144-2160.

Vesković, S., Stević Ž., Stojić, G., Vasiljević, M. (2018). Evaluation of the railway management model by using a new integrated model DELPHI-SWARA-MABAC. decision making: Applications in Management and Engineering, 1, 34-50.

Wang, H., Smarandache, F., Zhang, Y.Q., Sunderraman, R. (2005). Interval Neutrosophic Sets and Logic: Theory and Applications in Computing. Phoenix, Hexis, AZ, USA.

Wang, H., Smarandache, F., Zhang, Y.Q., Sunderraman, R. (2010). Single valued neutrosophic sets. Multispace Multistruct, 410-413.

Wang, J., Wei, G.W., Lu, M. (2018a). An extended VIKOR method for multiple criteria group decision making with triangular fuzzy neutrosophic numbers. Symmetry-Basel, 10.

Wang, J., Wei, G.W., Lu, M. (2018b). TODIM method for multiple attribute group decision making under 2-tuple linguistic neutrosophic environment. Symmetry-Basel, 10.

Wang, J., Wei, G.W., Lu, M. (2018c). TODIM method for multiple attribute group decision making under 2-tuple linguistic neutrosophic environment. Symmetry-Basel, 10, 486.

Wang, J., Wei, G.W., Wei, Y. (2018d). Models for Green supplier selection with some 2-tuple linguistic neutrosophic number Bonferroni mean operators. Symmetry-Basel, 10.

Wang, J., Tang, X.Y., Wei, G.W. (2018e). Models for multiple attribute decision-making with dual generalized single-valued neutrosophic bonferroni mean Operators. Algorithms, 11.

Wang, J., Gao, H., Wei, G.W., Wei, Y. (2019a). Methods for multiple-attribute group decision making with qrung interval-valued orthopair fuzzy information and their applications to the selection of Green suppliers. Symmetry-Basel, 11, 56.

Wang, R., Wang, J., Gao, H., Wei, G.W. (2019b). Methods for MADM with picture fuzzy muirhead mean operators and their application for evaluating the financial investment risk. Symmetry-Basel, 11, 6. 
Wei, G.W. (2018). TODIM method for picture fuzzy multiple attribute decision making. Informatica, 29, 555566.

Wei, G.W. (2019). Pythagorean fuzzy hamacher power aggregation operators in multiple attribute decision making. Fundamenta Informaticae, 166, 57-85.

Wei, G.W., Gao, H. (2018). The generalized dice similarity measures for picture fuzzy sets and their applications. Informatica, 29, 107-124.

Wei, G., Wei, Y. (2018a). Some single-valued neutrosophic dombi prioritized weighted aggregation operators in multiple attribute decision making. Journal of Intelligent \& Fuzzy Systems, 35, 2001-2013.

Wei, G.W., Wei, Y. (2018b). Similarity measures of Pythagorean fuzzy sets based on the cosine function and their applications. International Journal of Intelligent Systems, 33, 634-652.

Wei, G.W., Zhang, Z.P. (2019). Some single-valued neutrosophic Bonferroni power aggregation operators in multiple attribute decision making. Journal of Ambient Intelligence and Humanized Computing, 10, 863 882.

Wei, Y., Liu, J., Lai, X., Hu, Y. (2017). Which determinant is the most informative in forecasting crude oil market volatility: fundamental, speculation, or uncertainty? Energy Economics, 68, 141-150.

Wei, G.W., Alsaadi, F.E., Hayat, T., Alsaedi, A. (2018a). Bipolar fuzzy hamacher aggregation operators in multiple attribute decision making. International Journal of Fuzzy Systems, 20, 1-12.

Wei, G.W., Gao, H., Wang, J., Huang, Y.H. (2018b). Research on risk evaluation of enterprise human capital investment with interval-valued bipolar 2-tuple linguistic information. Ieee Access, 6, 35697-35712.

Wei, G.W., Gao, H., Wei, Y. (2018c). Some q-rung orthopair fuzzy Heronian mean operators in multiple attribute decision making. International Journal of Intelligent Systems, 33, 1426-1458.

Wei, G.W., Garg, H., Gao, H., Wei, C. (2018d). Interval-valued Pythagorean fuzzy Maclaurin symmetric mean operators in multiple attribute decision making. Ieee Access, 6, 67866-67884.

Wei, G.W., Wei, C., Gao, H. (2018e). Multiple attribute decision making with interval-valued bipolar fuzzy information and their application to emerging technology commercialization evaluation. IEEE Access, 6, 60930-60955.

Wei, Y., Yu, Q., Liu, J., Cao, Y. (2018f). Hot money and China's stock market volatility: further evidence using the GARCH-MIDAS model. Physica A: Statistical Mechanics and Its Applications, 492, 923-930.

Wei, G.W., Wei, C., Wang, J., Gao, H., Wei, Y. (2019). Some q-rung orthopair fuzzy maclaurin symmetric mean operators and their applications to potential evaluation of emerging technology commercialization. International Journal of Intelligent Systems, 34, 50-81.

Wu, Q., Wu, P., Zhou, L.G., Chen, H.Y., Guan, X.J. (2018a). Some new Hamacher aggregation operators under single-valued neutrosophic 2-tuple linguistic environment and their applications to multi-attribute group decision making. Computers \& Industrial Engineering, 116, 144-162.

Wu, S.J., Wang, J., Wei, G.W., \& Wei, Y. (2018b). Research on construction engineering project risk assessment with some 2-tuple linguistic neutrosophic hamy mean operators. Sustainability, 10.

Xu, D.S., Wei, C., Wei, G.W. (2017). TODIM method for single-valued neutrosophic multiple attribute decision making. Information, 8.

Xue, Y.X., You, J.X., Lai, X.D., Liu, H.C. (2016). An interval-valued intuitionistic fuzzy MABAC approach for material selection with incomplete weight information. Applied Soft Computing, 38, 703-713.

Ye, J. (2014). A multicriteria decision-making method using aggregation operators for simplified neutrosophic sets. Journal of Intelligent \& Fuzzy Systems, 26, 2459-2466.

Yu, S.M., Wang, J., Wang, J.Q. (2017). An interval type-2 fuzzy likelihood-based MABAC approach and its application in selecting hotels on a tourism website. International Journal of Fuzzy Systems, 19, 47-61.

Zadeh, L.A. (1965). Fuzzy Sets. Information and Control, 8, 338.

Zavadskas, E.K., Antucheviciene, J., Saparauskas, J., Turskis, Z. (2013). MCDM methods WASPAS and MULTIMOORA: verification of robustness of methods when assessing alternative solutions. Economic Computation and Economic Cybernetics Studies and Research, 47, 5-20.

Zhang, S.Q., Gao, H., Wei, G.W., Wei, Y., Wei, C. (2019). Evaluation based on distance from average solution method for multiple criteria group decision making under picture 2-tuple linguistic environment. Mathematics, 7, 243. 
P. Wang is a current master student with Institute of Technology, Sichuan Normal University, Chengdu, 610101, PR China.

J. Wang is a current master student with School of Business at Sichuan Normal University, Chengdu, 610101, PR China. He has published more than 10 papers in journals, such as International Journal of Intelligent Systems, Journal of Intelligent and Fuzzy Systems, IEEE Access, Mathematics, Symmetry-Base. He is currently interested in multiple attribute decision making and computing with words.

G. Wei has an MSc and a PhD degree in applied mathematics from SouthWest Petroleum University, business administration from school of Economics and Management at SouthWest Jiaotong University, China, respectively. From May 2010 to April 2012, he was a postdoctoral researcher with the School of Economics and Management, Tsinghua University, Beijing, China. He is a professor in the School of Business at Sichuan Normal University. He has published more than 100 papers in journals, books and conference proceedings including journals such as Omega, Decision Support Systems, Expert Systems with Applications, Applied Soft Computing, Knowledge and Information Systems, Computers \& Industrial Engineering, Knowledge-based Systems, International Journal of Intelligent Systems, International Journal of Uncertainty, Fuzziness and Knowledge-Based Systems, International Journal of Computational Intelligence Systems, International Journal of Machine Learning and Cybernetics, Fundamenta Informaticae, Informatica, Kybernetes, International Journal of Knowledge-based and Intelligent Engineering Systems and Information: An International Interdisciplinary Journal. He has published 1 book. He has participated in several scientific committees and serves as a reviewer in a wide range of journals including Computers \& Industrial Engineering, International Journal of Information Technology and Decision Making, Knowledge-based Systems, Information Sciences, International Journal of Computational Intelligence Systems and European Journal of Operational Research. He is currently interested in aggregation operators, decision making and computing with words.

C. Wei has an MSc degree in applied mathematics from SouthWest Petroleum University. Now, he is a PhD student with School of Statistics, Southwestern University of Finance and Economics, Chengdu, 611130, PR China. He has published more than 10 papers in journals, such as International Journal of Intelligent Systems, Journal of Intelligent and Fuzzy Systems, IEEE Access, Mathematics, Information. He is currently interested in aggregation operators, decision making and computing with words.

Y. Wei has an MSc and a PhD degree in management science and engineering from Southwest Jiaotong University, China. He is a professor in the School of Finance at Yunnan University of Finance and Economics. He has published more than 120 papers in journals, books and conference proceedings including journals such as Journal of Forecasting, Journal of Banking \& Finance, Empirical Economics, Energy Economics, Economic Modelling, Applied Economics Letters, International Review of Economics \& Finance. He has published two books (in Chinese). He has participated in several scientific committees and serves as a reviewer in a wide range of journals including Energy Economics, 
Journal of Forecasting, Emerging Market Finance and Trade, Physica A. He is currently interested in volatility modelling and forecasting in financial and energy markets, density forecasting and decision making in financial markets. 